\title{
Interfering with the Actin Network and Its Effect on Long-Term Potentiation and Synaptic Tagging in Hippocampal CA1 Neurons in Slices In Vitro
}

\author{
Binu Ramachandran and Julietta U. Frey \\ Department of Neurophysiology, Leibniz Institute for Neurobiology, 39118 Magdeburg, Germany
}

\begin{abstract}
Long-term potentiation (LTP) is a cellular correlate for memory formation, which requires the dynamic changes of the actin cytoskeleton. As shown by others, the polymerization of the actin network is important for early stages of LTP. Here, we investigated the role of actin dynamics in synaptic tagging and particularly in the induction of protein synthesis-dependent late-LTP in the CA1 region in hippocampal slices in vitro. We found that the inhibition of actin polymerization affects protein synthesis-independent early-LTP, prevents late-LTP, and interferes with synaptic tagging in apical dendrites of hippocampal CA1. The transformation of early-LTP into late-LTP was blocked by the application of the structurally different actin polymerization inhibitors latrunculin A or cytochalasin D. We suggest that the actin network is required for early "housekeeping" processes to induce and maintain early-LTP. Furthermore, inhibition of actin dynamics negatively interacts with the setting of the synaptic tagging complex. We propose actin as a further tag-specific molecule in apical CA1 dendrites where it is directly involved in the tagging/capturing machinery. Consequently, inhibition of the actin network prevents the interaction of tagging complexes with plasticity-related proteins. This results in the prevention of late-LTP by inhibition of the actin network during LTP induction.
\end{abstract}

\section{Introduction}

Changes in synaptic efficacy are considered as basic processes of information storage in the brain. Currently, there are only two cellular phenomena which could underlie such prolonged changes called long-term potentiation (LTP) and long-term depression (LTD) (Matthies et al., 1990; Bear and Malenka, 1994; Frey and Frey, 2008). Both, LTP and LTD show distinct temporal stages such as a protein synthesis-independent early phase (earlyLTP, early-LTD) and a protein synthesis-dependent late phase (late-LTP, late-LTD). LTP/LTD maintenance requires the synthesis of plasticity-related proteins (PRPs) (Krug et al., 1984; Frey et al., 1988; Otani et al., 1989; Nguyen and Kandel, 1996; Manahan-Vaughan et al., 2000). How PRPs interact only with activated ("tagged") synapses expressing LTP or LTD is fundamental to synapse-specificity of the process and can be explained by the "synaptic tagging" hypothesis (Frey and Morris, 1997, 1998a,b). Specific stimulation transiently sets a "tag" at activated synapses and induces PRP synthesis. If both tags and PRPs are available within a specific time window, they can interact, thus transforming a normally transient early-LTP (E-LTP) into lateLTP (L-LTP) (Frey and Morris, 1998a; Frey and Frey, 2008).

Received April 30, 2009; revised July 31, 2009; accepted Aug. 24, 2009.

This work was supported by Deutsche Forschungsgemeinschaft Grant 1034-7 and Sonderforschungsbereich Grant 779-B4 to J.U.F. We are grateful to Dr. Jeffrey Vernon for his very helpful input on this manuscript and to Dr. Sajikumar Sreedharan for his help to train B.R. in in vitro slice techniques, as well as for his technical support and discussions. We are also very grateful to Diana Koch for her excellent technical assistance.

Correspondence should be addressed to Dr. Julietta U. Frey, Department of Neurophysiology, Leibniz Institute for Neurobiology, Brenneckestrasse 6, 39118 Magdeburg, Germany. E-mail: frey@ifn-magdeburg.de.

D01:10.1523/JNEUROSCI.2045-09.2009

Copyright $\odot 2009$ Society for Neuroscience $\quad 0270-6474 / 09 / 2912167-07 \$ 15.00 / 0$
The actin network plays an important, multifunctional role in synaptic function as well as during plasticity and, specifically, in hippocampal LTP. Actin depolymerization blocks both functional as well as structural plasticity (Krucker et al., 2000; Fukazawa et al., 2003; Lisman, 2003; Matsuzaki et al., 2004; Okamoto et al., 2004; Chen et al., 2007; Tanaka et al., 2008). The actin network is associated with a rapid and persistent reorganization of the spine cytoskeleton (Fischer et al., 1998; Okamoto et al., 2004; Lin et al., 2005). Furthermore, an increase in F-actin content and structural changes within dendritic spines during LTP induction were described previously (Moshkov and Petrovskaia, 1983; Fifková, 1985; Pak et al., 2001; Fukazawa et al., 2003; Lisman, 2003; Okamoto et al., 2004, 2007; Chen et al., 2007), and it was shown that the actin cytoskeleton mediates exocytosis and endocytosis of AMPAreceptors at postsynaptic sites (Malinow et al., 2000; Sheng and Lee, 2001). Actin polymerization is also involved in the regulation of the synthesis of protein kinase Mzeta (PKMzeta), which enhances synaptic efficacy during late-LTP by recruiting more postsynaptic AMPA receptors (Serrano et al., 2005; Ling et al., 2006). PKMzeta has been identified as a PRP required for lateLTP in the apical CA1 dendrites (Sajikumar et al., 2005b). Because the actin network is directly involved in local protein trafficking within the dendritic spine (Kaech et al., 1996; Langford and Molyneaux, 1998), it could also exert its action during the capture of PRPs at the tagged synapse. Here, we further investigate the possible specific action of actin network function on processes required for late-LTP in the hippocampal CA1 in vitro. We suggest that the actin network interacts with late-LTP and specifically with the PRP-capturing process at synaptic tags (Frey and Morris, 1998a; Martin and Kosik, 2002). By using structur- 
ally different actin assembly inhibitors, latrunculin A or cytochalasin D, we show that actin function is involved in early-LTP, and it is essential for lateLTP by interacting with the synaptic tagging machinery.

\section{Materials and Methods}

All experiments were performed in accordance with the European Community Council Directive of 24 November 1986 (86/609/EEC). We also certify that formal approval to conduct the experiments has been obtained from the animal subject review board of our institution/ local government which can be provided upon request. All efforts were made to minimize the number of animals used and their suffering.

Slice preparation and electrophysiology. Transverse hippocampal slices $(112 ; 400 \mu \mathrm{m})$ were prepared from 112 7-week-old male Wistar rats as described previously (Frey et al., 1988; Sajikumar et al., 2005a). The rats were killed rapidly by a single blow to the back of the neck using a metallic rod (cervical dislocation) and immediately decapitated. Slices were incubated in an interface chamber at $32^{\circ} \mathrm{C}$, and the incubation medium [artificial cerebrospinal fluid (ACSF)] contained (in mM) $124 \mathrm{NaCl}, 4.9$ $\mathrm{KCl}, 1.2 \mathrm{KH}_{2} \mathrm{PO}_{4}, 2.0 \mathrm{MgSO}_{4}, 2.0 \mathrm{CaCl}_{2}, 24.6$ $\mathrm{NaHCO}_{3}, 10.0$ D-glucose. The carbogen consumption was $32 \mathrm{~L} / \mathrm{h}$, and the flow rate of ACSF was $0.7 \mathrm{ml} / \mathrm{min}$ (the entire procedure from killing the animal after placing the slices into the recording chamber took $\sim 3-5 \mathrm{~min}$ ). In all experiments, two monopolar lacquer-coated, stainless-steel electrodes (5M $\Omega$; AM-Systems) were positioned within the stratum radiatum of the CA1 region for stimulating two separate independent synaptic inputs, S1 and S2. For recording the field EPSP (measured as its slope function) and the population spike (PS) amplitude, two electrodes (resistance, $5 \mathrm{M} \Omega$; AMSystems) were placed in the CA1 apical dendritic and cell body layer, respectively (Fig. 1A). Signals were amplified by a custom-made Leibniz Institute for Neurobiology amplifier. The signals were digitized using a CED1401Plus analog-to-digital converter (CED) and analyzed with custom-made software (PWIN). Slices were preincubated for at least $4 \mathrm{~h}$, which is critical for reliable long-lasting recordings of late-LTP (for more details, see Sajikumar et al. (2005a).

After the preincubation period, the test stimulation strength was determined for each input to elicit a PS of $40 \%$ of its maximal amplitude for the control input and $25 \%$ for inputs in which LTP was induced. The PS amplitude and the slope of the field EPSP were monitored on-line. Baseline was recorded using test stimuli consisting of four biphasic constant current pulses ( $\mathrm{f}=0.2 \mathrm{~Hz}$; impulse width, $0.1 \mathrm{~ms}$ per half wave; averaged on-line) per time point every $15 \mathrm{~min}$ for a minimum period of $1 \mathrm{~h}$ before LTP induction. In experiments with induction of early-LTP, a weak tetanization protocol (WTET) consisting of a single $100 \mathrm{~Hz}$ train (21 biphasic constant current pulses; pulse width duration, $0.2 \mathrm{~ms}$ per polarity; stimulus intensity for tetanization: PS threshold). Late-LTP was induced using three stimulus trains of 100 pulses ["strong" tetanus (STET), $\mathrm{f}=$ $100 \mathrm{~Hz}$; impulse duration, $0.2 \mathrm{~ms} /$ polarity; intertrain interval, $10 \mathrm{~min}$ ]. Four biphasic constant-current pulses ( $\mathrm{f}=0.2 \mathrm{~Hz}$; impulse duration, 0.1 ms per polarity) were used for baseline recordings and testing $1,3,5,11$, $15,21,25,30 \mathrm{~min}$ after tetanus and then every $15 \mathrm{~min}$ up to $6 \mathrm{~h}$, i.e., the end of the experiment.

Pharmacological substances. Latrunculin A (Calbiochem), dissolved in ACSF and $0.1 \%$ dimethyl sulfoxide (DMSO) to prevent actin polymerization, was used at a concentration of $0.1 \mu \mathrm{M}$ (Kim and Lisman, 1999). A
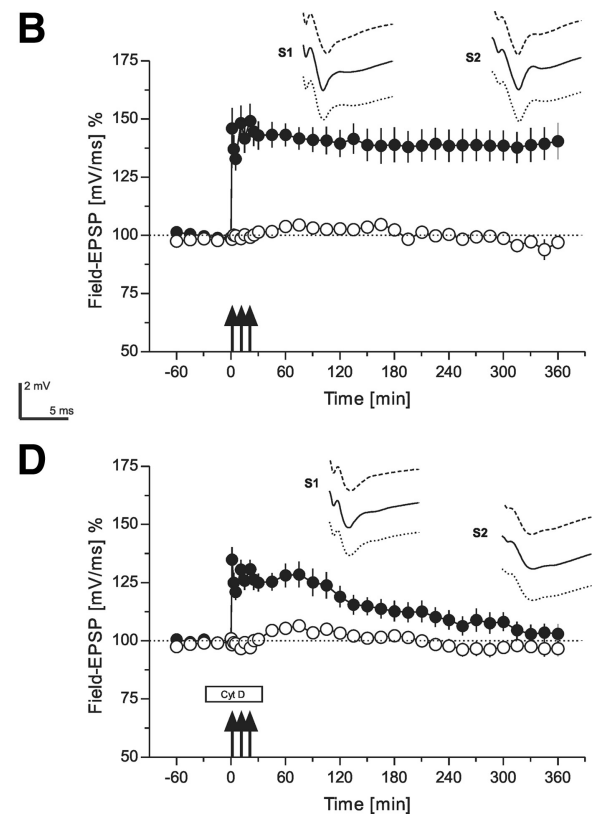

Figure 1. The role of the actin network in long-term potentiation, i.e., late-LTP (time courses of field EPSP recordings). 作 2 in region. The location of recording electrodes for the PS as well as the field EPSP from

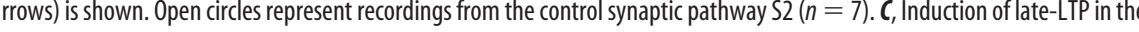
was applied 30 min before STET, and it was washed-out 30 min after the first tetanization (open circles: controlinput S2). Analog dotted line) after tetanization of input $\$ 1$. The analog traces for $\$ 2$ were recorded at the same time points, however, without tetanization. Calibration: $2 \mathrm{mV}$, $5 \mathrm{~ms}$. Arrows indicate the time point of tetanization of synaptic input S1 (STET are symbolized as 3 arrows). Filled boxes represent drug application. Lat A, latrunculin A; Cyt D, cytochalasin D.

structurally different actin polymerization inhibitor, cytochalasin D (Calbiochem) was used at a concentration of $0.1 \mu \mathrm{M}$. Cytochalasin D was dissolved in ACSF and 0.1\% DMSO. Anisomycin (Sigma), a reversible protein synthesis inhibitor, was used at a concentration of $25 \mu \mathrm{M}$ (a concentration that blocked at least $85 \%$ of $\left[{ }^{3} \mathrm{H}\right]$ leucine incorporation into hippocampal slices) (Frey et al., 1991). Anisomycin was dissolved in ACSF and 0.1\% DMSO.

Statistical analysis of electrophysiological data. Averaged values of the PS amplitude and the slope function of the field EPSP (expressed as their percentage change to baseline recordings within the $1 \mathrm{~h}$ baseline recording) per time point were subjected to statistical analysis by the nonparametric Wilcoxon signed rank test, when compared within one group or the Mann-Whitney $U$ test when data were compared between groups ( $p<0.05$ were considered as being statistically significantly different).

\section{Results}

\section{Actin network and L-LTP}

Induction of late-LTP by STET of synaptic input S1 (Fig. $1 B$, filled circles) resulted in a persistent, statistically significant form of LTP with a duration of at least $6 \mathrm{~h}$, when compared with the values in a control baseline pathway $\mathrm{S} 2$ (Fig. $1 B$, open circles) $(U$ test, $p<0.05$ ) or its own baseline values before TET (Wilcoxon, $p<0.05)$. The control input S2, without STET, remained stable at baseline levels for the entire experimental session (Fig. 1B, open circles). In the next set of experiments (Fig. 1C), we investigated the effect of latrunculin A on late-LTP and, therefore, the drug was applied during STET, i.e., during late-LTP induction. As shown in Figure $1 C$ (filled circles), application of 

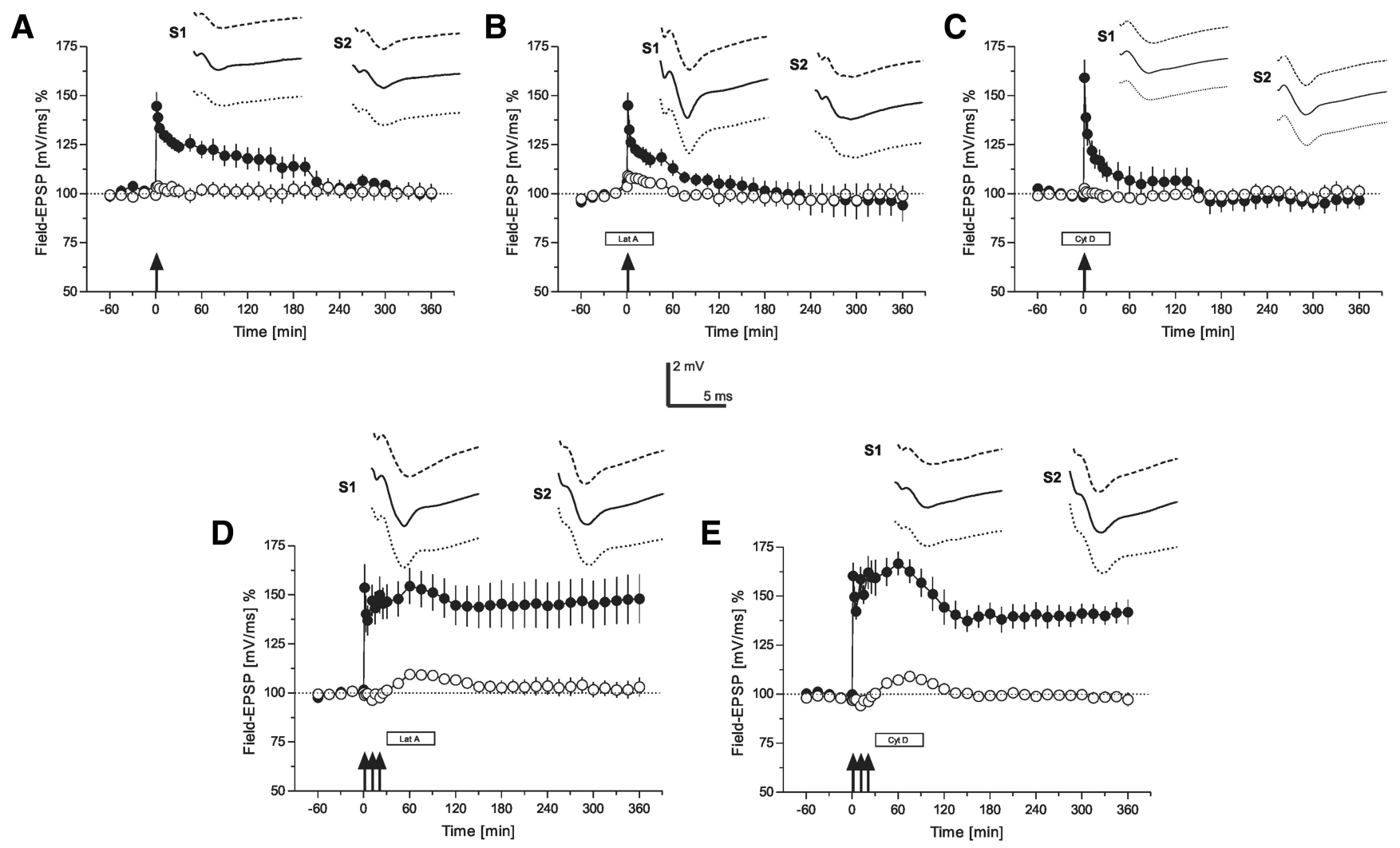

$\bigsqcup^{2 \mathrm{mV}} \mathrm{ms}$

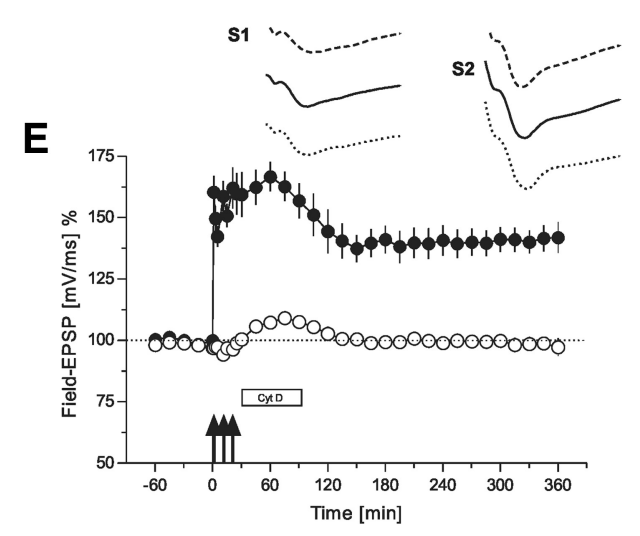

Figure 2. The role of the actin network for early-LTP (time courses of field EPSP recordings). $A$, Time course of the slope of field EPSP after the induction of early-LTP by WTET (indicated by the single arrow) in S1 (filled circles). The control pathway $\$ 2$ (open circles) remained stable throughout the experiment $(n=7)$. $\boldsymbol{B}$, The influence of latrunculin A on early-LTP: the drug was applied 30 min before WTET of $S 1$ (filled circles; open circles represent the time course of the control input $S 2 ; n=7$ ). $C$, Similar to $B$, but now instead of latrunculin $A$, cytochalasin D was applied 30 min before WTET of $S 1$ (filled circles; open circles represent the time course of the control input $S 2 ; n=7$ ). $\boldsymbol{D}$, The effect of latrunculin A on late-LTP if applied 30 min after STET in S1 (filled circles). 0 pen circles represent the time course of the control input $S 2(n=6)$. $\boldsymbol{E}$, Similar to $\boldsymbol{D}$, but instead of latrunculin $A$, now cytochalasin $D$ was applied $(n=7)$. The time points of the analog traces as well as the calibration as in Figure 1. Arrows indicate the time point of tetanization (STET are symbolized as 3 arrows, WTET by a single arrow, were appropriate). Boxes as in Figure 1.

latrunculin A (30 min before until $30 \mathrm{~min}$ after STET in input S1) nonsignificantly affected the level of potentiation immediately after tetanization and prevented the expression of lateLTP. The potentiation was only statistically significantly different from potentials of the control pathway until $4 \mathrm{~h}$ and $15 \mathrm{~min}(U$ test, $p<0.05$ ). Control responses obtained from S2 (Fig. $1 C$, open circles) remained relatively stable at baseline levels. To confirm these results, we used another actin inhibitor, cytochalasin D. Application of cytochalasin D during late-LTP induction, i.e., from 30 min before until $30 \mathrm{~min}$ after the first tetanus in input $\mathrm{S} 1$ (Fig. 1D, filled circles) again nonsignificantly affected the level of the initial potentiation and also prevented the late form of LTP. Baseline values were reached already after $2 \mathrm{~h}$ and $45 \mathrm{~min}$ ( $U$ test). Control responses in S2 (Fig. 1D, open circles) again remained relatively stable at baseline levels. Then, we investigated whether the actin network was also required for a transient, i.e., early-LTP.

\section{Actin network and E-LTP}

Induction of early-LTP in synaptic input S1 by WTET (Fig. $2 A$, filled circles) resulted in a transient form of LTP, which was statistically significantly different from control values measured in S2 (Fig. $2 A$, open circles) for up to $90 \mathrm{~min}(U$ test, $p<0.05)$ or for $2 \mathrm{~h}$ when compared with pretetanization levels (Wilcoxon, $p<0.05$ ). The control input S2, without application of highfrequency stimulation, remained again stable at baseline levels for the entire experimental session (Fig. $2 \mathrm{~A}$, open circles). Next, we applied latrunculin A during the induction of early-LTP (Fig. 2B, $\mathrm{S} 1$, filled circles). Interestingly, the drug even affected the immediate maintenance of early-LTP when compared with untreated early-LTP (Fig. $2 \mathrm{~A}$, filled circles) $60 \mathrm{~min}$ after tetanization, suggesting an immediate effect of the actin network on the early potentiation, i.e., early-LTP $(U$ test, $p<0.05)$. The control input S2 remained relatively stable at baseline levels for the entire experimental session (Fig. $2 B$, open circles). Similar results were obtained if instead of latrunculin A, cytochalasin D was applied (Fig. 2C). Again, early-LTP induction was already slightly affected by the drug (Fig. 2C, S1, filled circles) without effects of the drug on baseline recordings (Fig. 2C, S2, open circles). Early-LTP in S1 under the influence of cytochalasin D was statistically significantly different from control recordings in S2 only up to 45 min after tetanization $(U$ test, $p<0.05)$. In the following experiment, we applied latrunculin A 30 min after STET in input S1 (Fig. 2D, filled circles) for a duration of $1 \mathrm{~h}$. No effect on the maintenance of LTP could be observed. Also, the control input (Fig. 2D, open circles) remained relatively stable at baseline values, although a small statistically significant but transient "wash-in" effect was seen from 45 to 120 min after STET (Wilcoxon, $p<0.05)$. When cytochalasin $\mathrm{D}$ was applied $30 \mathrm{~min}$ after STET instead of latrunculin A (Fig. 2E), late-LTP was also not prevented in $S 1$, although a statistically nonsignificant reduction of late-LTP after the application of cytochalasin D was observed (Fig. $2 E$, filled circles) and a similar transient wash-in effect was observed in input S2 (statistically different from 45 to $75 \mathrm{~min}$ 

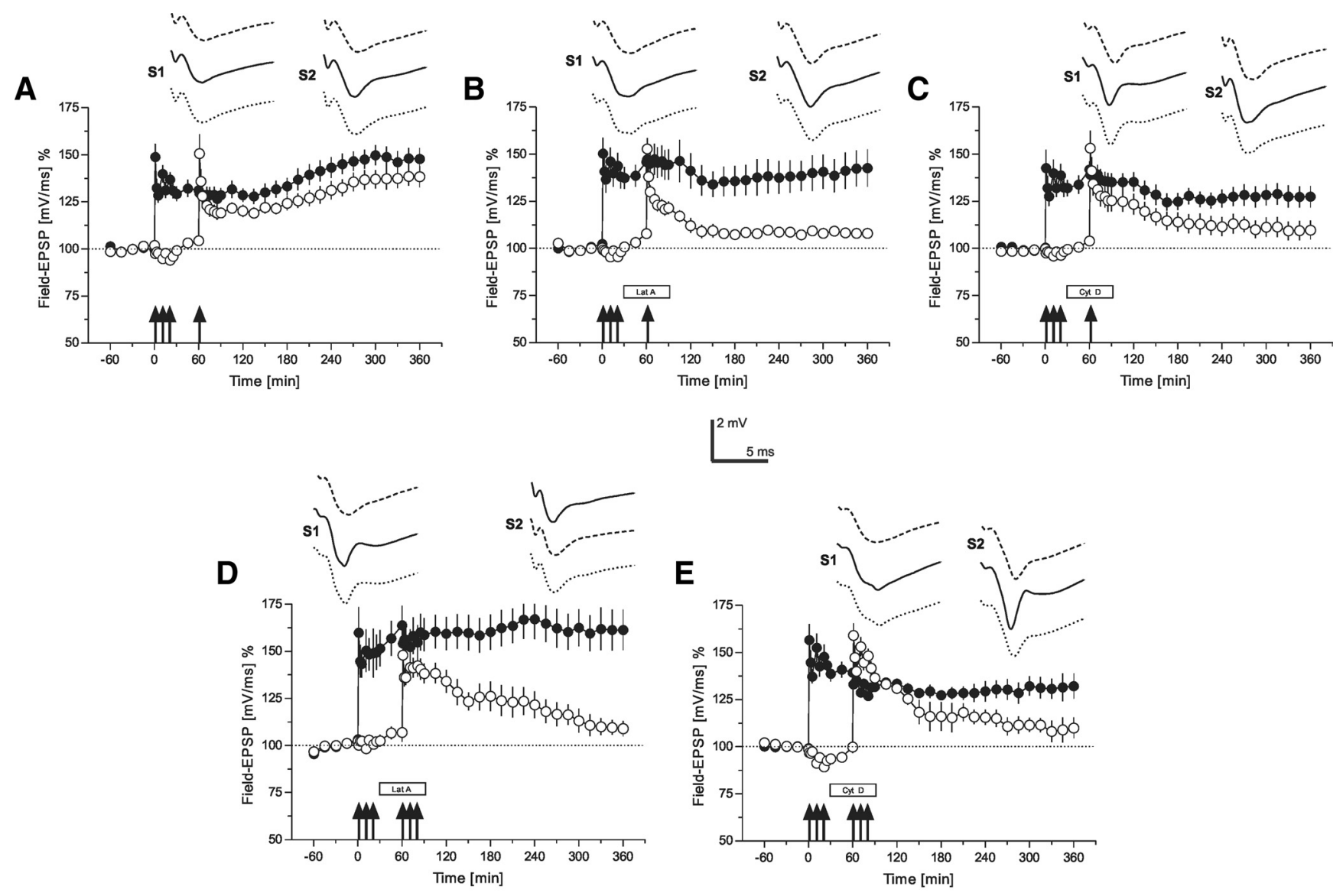

Figure 3. The role of the actin network for synaptic tagging (time courses of field EPSP recordings) — "strong-tetanization before weak-tetanization" and "strong before strong." $\boldsymbol{A}$, This panel represents a control experiment verifying synaptic tagging in our preparation. The time course of the slope of the field EPSP after STET of input S1 (filled circles; the tetanization is symbolized by the arrows) is presented. One hour after STET to S1, a WTET to S2 (open circles) was applied. The normally induced early-LTP in S2 was transformed into late-LTP by the previous induction of late-LTP in $S 1(n=7)$. $\boldsymbol{B}$, The time course of the same tagging experiment as in $\boldsymbol{A}$ is presented, however, with the application of latrunculin A during the time of WTET to S2 (open circles; box represents drug application). Latrunculin A was applied 30 min before and until 30 min after WTET of $S 2(n=7)$. Synaptic tagging in $S 2$ was thus prevented. $\boldsymbol{C}$, A similar experiment as in $\boldsymbol{B}$; however, instead of latrunculin A, now cytochalasin D was applied ( $n=7$ ). Again, synaptic tagging in $\mathbf{S 2}$ was prevented by the drug. $\boldsymbol{D}$, The same procedure as in $\boldsymbol{B}$; however, now STET was also applied to S2 (open circles) in the presence of latrunculin A $(n=6)$. Late-LTP in S2 was also prevented by the drug regardless of the previous STET and induction of late-LTP in S1 (filled circles). $E$, The same procedure as in $\boldsymbol{D}$ with the exception that instead of latrunculin A now cytochalasin D was applied $(n=6)$. Again, late-LTP in 22 was prevented. The time points of the analog traces as in Figure 1; however, here $\mathrm{S} 2$ was also tetanized. Therefore, the traces of $\mathrm{S} 1$ and $\mathrm{S} 2$ represent representative examples $30 \mathrm{~min}$ before (dashed line), $30 \mathrm{~min}$ (solid line), and $6 \mathrm{~h}$ (dotted line) after tetanization of the adequate input. Symbols and calibration as in Figure 2.

after STET; Wilcoxon, $p<0.05)$. Thus, when the actin blockers were applied after late-LTP induction, the maintenance of lateLTP was not influenced anymore.

\section{Actin network and processes of synaptic tagging \\ "Strong before weak"}

As seen from the last experiments, actin network function must be intact during or shortly after the induction of LTP, i.e., within a short time window, which reminded us on processes of synaptic tagging. Thus, in the first set of control experiments, we reconfirmed the tagging process in our preparation-late-LTP was induced in synaptic input S1 by STET (Fig. 3A, filled circles), and 60 min thereafter, an early form of LTP was induced in synaptic input S2 by WTET (Fig. 3A, open circles). As shown in Figure 3A, the normally transient early-LTP in S2 was transformed into lateLTP by the previous induction of late-LTP in S1, supporting our earlier data that the strong input $\mathrm{S} 1$ activates its synapse-specific tag complex and the synthesis of synapse-unspecific PRPs. If within an effective time window an independent input S2 was activated by a WTET, which sets its synapse-specific tags but was unable to induce its synthesis of PRPs, then this input S2 could benefit from the PRPs synthesized by the STET in S1. The S2-tag complex could then capture the PRPs and thus was able to transform its normally early-LTP into late-LTP by means of the availability of its own synapse-specific S2-tag complex and the synapse-unspecific PRPs (Frey and Morris, 1997, 1998a,b). Next, we investigated whether the actin network was involved in processes of synaptic tagging. We induced late-LTP in S1 (Fig. 3B, filled circles), which was statistically significant up to $6 \mathrm{~h}$ (Wilcoxon, $p<$ 0.05 ), and $60 \mathrm{~min}$ after STET, early-LTP was induced in S2 by a WTET, however, now in the presence of latrunculin A (Fig. 3B, open circles). Latrunculin A was applied from 30 min before until $30 \mathrm{~min}$ after the induction of early-LTP in input S2. In contrast to normal tagging as shown in Figure $3 \mathrm{~A}$, latrunculin A prevented it, i.e., E-LTP in $\mathrm{S} 2$ was not transformed into late-LTP by the previous late-LTP induction in S1. A potentiation in S2 was only detected from 61 to $285 \mathrm{~min}$, i.e., from $1 \mathrm{~min}$ to $2 \mathrm{~h}$ and $45 \mathrm{~min}$ after WTET to S2 (Wilcoxon, $p<0.05$ ). To confirm this result, we repeated this set of experiments, but instead of latrunculin $A$, we now used cytochalasin D. Also, the latter prevented the transformation of early-LTP into late-LTP (Fig. 3C, open circles) (a po- 

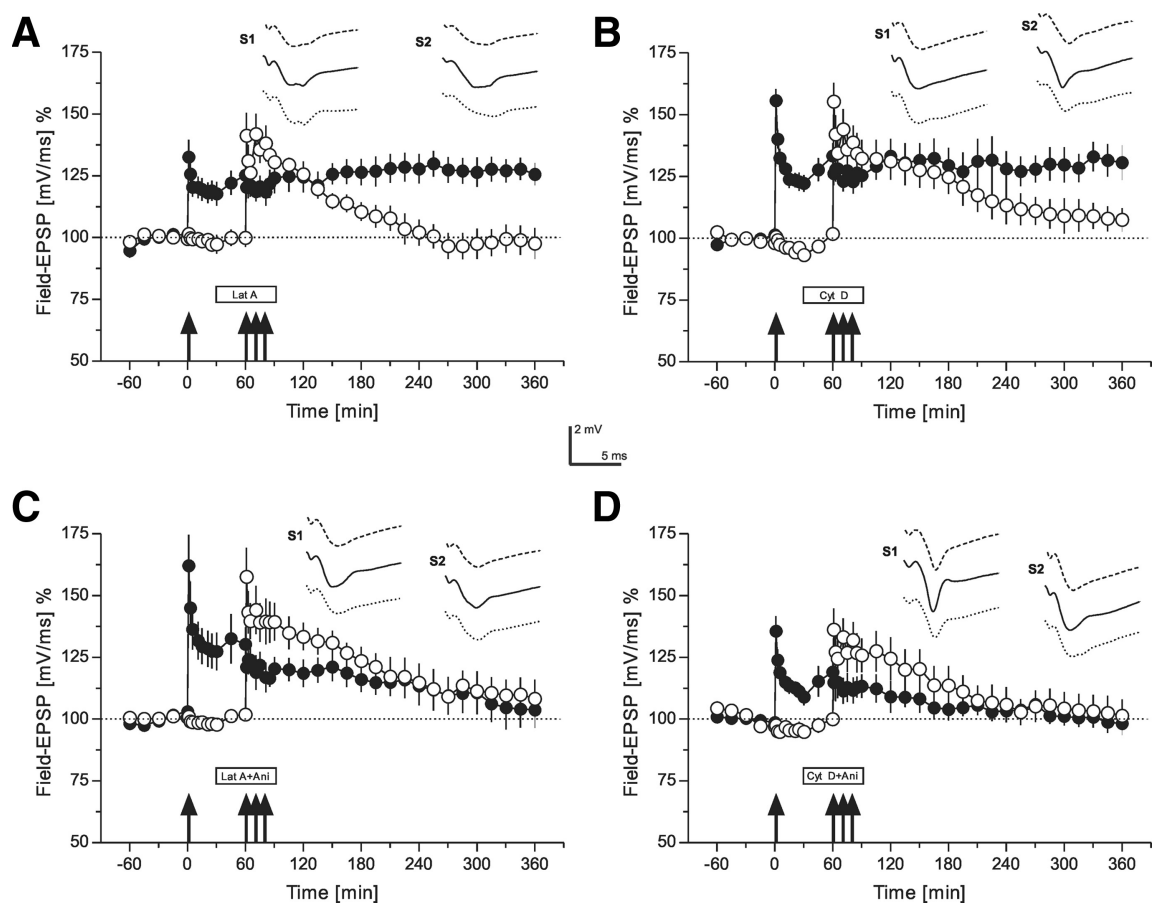

Figure 4. The role of the actin network for synaptic tagging (time courses of field EPSP recordings) — "weak-tetanization before strong-tetanization." A, A weak before strong protocol was used (Frey and Morris, 1998b). In S1 (filled circles), WTET was applied at the time point indicated by the single arrow. One hour later, a STET was applied to input S2 (open circles). However, now latrunculin A was applied (30 min before STET to S2 until 30 min after STET to S2). Although, late-LTP in S2 was prevented, the tagging process was observed in S1, i.e., the normally early-LTP was transformed into late-LTP in S1 by the STET of S2 even under inhibition of actin polymerization $(n=6)$. $\boldsymbol{B}$, A similar weak before strong experiment as in $\boldsymbol{A}$ with the exception that instead of latrunculin A the structurally different actin polymerization inhibitor cytochalasin D was applied. Again, late-LTP in S2 was blocked; however, tagging in $\mathbf{S 1}$ took place $(n=6)$. C, A similar experimental design as in $\boldsymbol{A}$ with the exception that together with latrunculin $A$, the reversible protein synthesis inhibitor anisomycin was applied $(n=6)$. All late forms or LTP were prevented. D, The same as in $\boldsymbol{C}$ with the exception that instead of latrunculin A, cytochalasin D was applied together with anisomycin. Again, all late forms of LTP were blocked $(n=6)$. The time points of the analog traces as well as symbols and calibration as in Figure 3. Ani, Anisomycin.

tentiation in S2 was also only detected until $2 \mathrm{~h}$ and 45 min after WTET to S2; Wilcoxon, $p<0.05$ ).

\section{"Strong before strong"}

In the next sets of experiments, we further investigated if the actin network prevents the setting of the tag complex or the synthesis of PRPs or whether it is required for both. Therefore, in the experiments presented in Figure 3D, we had now induced late-LTP in S1 by a STET (filled circles), which sets its S1-tags as well as activates the synthesis of PRPs. Thirty minutes after STET to S1, we applied latrunculin A for a duration of $1 \mathrm{~h}$. During this time, STET was now applied to S2 (Fig. 3D, open circles). Normally, late-LTP should be observed in S2, but latrunculin A blocked it from $3 \mathrm{~h}$ up to the end of the experiments, i.e., $5 \mathrm{~h}$ after STET to S2 (Wilcoxon, $p<0.05$ ). In Figure $3 E$, a similar experiment is shown; however, here the cytochalasin D was applied instead of latrunculin A. Again, late-LTP in S1 (Fig. 3E, filled circles) was not prevented, although partially reduced by cytochalasin D when compared with late-LTP in S1 and latrunculin A in Figure $3 D$. However, late-LTP in S2 (Fig. 3E, open circles) was also prevented by cytochalasin $\mathrm{D}$ (baseline levels were reached $5 \mathrm{~h}$ and 30 min after STET to S2; Wilcoxon test, $p<0.05$ ).

"Weak before strong"

The results above could suggest that the inhibition of the actin network might be more related to the inactivation of the tag complex instead of interfering with protein synthesis. To verify this, we then first induced early-LTP in S1 by a WTET (Fig. $4 A$, filled circles). This results in setting the tag without activating its own PRP synthesis in that input (Frey and Morris, 1997). Thirty minutes later, latrunculin A was applied for $1 \mathrm{~h}$. During that time, but 60 min after WTET in S1, STET to S2 was applied (Fig. $4 A$, open circles). STET in the presence of latrunculin A was unable to induce late-LTP in S2 (LTP returned to baseline levels after $2 \mathrm{~h}$ and $45 \mathrm{~min}$ in S2 after STET to this input in the presence of latrunculin A; Wilcoxon, $p<0.05$ ), but interestingly, early-LTP in S1 was transformed into late-LTP by this procedure. This result suggests that inhibition of the actin network directly interferes with the tag-setting machinery instead with the synthesis of PRPs, because the activated S1-tags must have been able to capture PRPs which were synthesized through the STET to S2 regardless of actin network inhibition. We confirmed this result by using also the second structurally different actin network blocker, cytochalasin D (Fig. 4B). Again, early-LTP in S1 (Fig. 4B, filled circles) was transformed into lateLTP if STET was applied to S2 $1 \mathrm{~h}$ after WTET to $\mathrm{S} 1$, even if the actin network was now blocked by cytochalasin D. Late-LTP in S2 (Fig. $4 B$, open circles), however, was prevented by the drug (after $4 \mathrm{~h}$ and 30 min after STET the potentials reached baseline levels; Wilcoxon, $p<0.05$ ). To verify that the transformation of earlyLTP into late-LTP in S1 was indeed achieved by the production of PRPs in S2, although under actin network inhibition, we repeated the experiments in Figure 4, $A$ and $B$, however, now with the coapplication of the actin network blockers with the reversible protein synthesis inhibitor anisomycin. As shown in Figure 4, $C$ and $D$ (filled circles), anisomycin prevented the transformation of early-LTP into late-LTP in S1, even if STET was applied to S2 in the presence of actin blockers and anisomycin, suggesting that the actin network interferes the setting of the tag machinery rather than with the synthesis of PRPs.

\section{Discussion}

In contrast to existing work from other laboratories, we were interested in studying the role of the actin network in prolonged forms of hippocampal LTP in apical CA1 dendrites. It has been shown that LTP is decreased or impaired when actin polymerization inhibitors are applied at the time of tetanization (Kim and Lisman, 1999; Krucker et al., 2000; Chen et al., 2007). These results are in accordance with ours obtained here, i.e., inhibition of actin polymerization negatively interfered with the induction of early-LTP (Fig. $3 B$ ) and prevented late-LTP (Fig. 1C,D). Interestingly, application of structurally different polymerization inhibitors, latrunculin $\mathrm{A}$ or cytochalasin D, $30 \mathrm{~min}$ after the induction of late-LTP by a STET, was unable to block LTP, suggesting an actin-dependent early mechanism for the induction and maintenance of LTP (Fig. 2 D, E). Previous work (Chen et al., 
2007) as well as our own data suggest that a relatively quick reassembly of the actin network is at least partially involved in the immediate maintenance of LTP (early-LTP) (Fig. $2 \mathrm{~B}, \mathrm{C}$ ) and is also required for late maintenance mechanisms (Fig. 1C,D). Although we do not yet fully understand the effect of actin polymerization prevention on early plasticity events, the active assembly of an actin-dependent cytoarchitecture is required for these processes. However, our major goal here was to investigate the possible involvement of the actin network on processes required for the induction and establishment of late-LTP, i.e., its role during synaptic tagging. If actin is involved in tagging, we asked if it is more related to the tagging machinery or to processes involved in the activation of the synthesis of PRPs. We had recently identified CaMKII as an LTP-specific tagging-related molecule, whereas PKMzeta is an LTP-specific PRP in apical CA1 dendrites (Sajikumar et al., 2005b, 2007). The question now arose if the role of the actin network could also be assigned to the tagging machinery or the PRP-synthesis machinery.

Our data show that late-LTP is prevented by the blockade of the actin network without having a significant effect on baseline recordings, suggesting that the activity of the actin network is not involved in neuronal or synaptic housekeeping processes. Our tagging experiments (Figs. 3,4) revealed that blockade of the actin network does not directly interfere with the synthesis of PRPs. If that were the case, then the WTET, inducing early-LTP at input S1 (Fig. 4A,B), should not have been transformed into late-LTP by the subsequent STET of an independent input S2 in the presence of the actin blockers. But early-LTP in S1 was in fact transformed into late-LTP under this condition (Fig. 4A,B). These results can only be explained by the following sequence of events. First, WTET to S1 sets the S1 synaptic tag (the WTET is unable to activate PRPs and would only normally result in earlyLTP). Subsequent STET to S2 would cause the synthesis of PRPs. The setting of S2-tags, however, was prevented by actin polymerization blockers. Therefore, input-unspecific PRPs induced through S2 could not be captured by the blocked S2-tags but only by the S1-tags, thus blocking late-LTP in S2 and transforming early-LTP into late-LTP in S1. We had shown earlier that the setting of LTP tags in the apical CA1 dendrites does not require protein synthesis (Frey and Morris, 1997, 1998b; Frey and Frey, 2008). Therefore, experiments presented in Figure 4, $C$ and $D$, suggest that actin is directly related to the tagging machinery rather than to PRP-related processes (but see below): STET of S2 can transform early-LTP into late-LTP in S1 even if the actin network is inhibited by the blockers (Fig. $4 A, B$ ). However, if in addition to the actin blockers a protein synthesis blocker was applied, this late-LTP transformation in S1 was also prevented (Fig. 4C,D). In the first scenario, synaptic tags in $\mathrm{S} 1$ were able to capture S2-induced PRPs and thus to transform S1 early-LTP into late-LTP. The actin blockers prevented the setting of the S2-tags; therefore, S2 could not benefit from its own induced PRP synthesis, thus revealing a prevention of late-LTP in S2. However, if S2-induced PRPs were also blocked, S1 also expressed only early-LTP even with set tags. Thus, we suggest that we have identified the actin network as a second tag-specific molecular complex, involved in the tagging machinery at least in the apical CA1 dendrites. However, we do not yet know if actin action is dedicated to the LTP-tag complex, as it is the case for CaMKII (Sajikumar et al., 2007) or whether it is also involved in a more general tagging machinery also required for LTD-tag complexes. Thus, it is feasible that the actin network guarantees the molecular/morphological basis for a tag-related housekeeping process, on top of which other key molecules like CaMKII or MAPK are only able to exert their action in synapses specifically marked either for LTP or LTD processes. Furthermore, the actin network could be involved in the PRP capture process itself by providing a distinct geometry for interactions of the kinases with the PRPs such as sculpting active synaptic zones or the whole synaptic spine, guaranteeing, for example, the activation or transport of PRPs into the spine or the transport of new AMPA-receptors to maintain LTP (Fukazawa et al., 2003; Lisman, 2003; Chen et al., 2007; Shoji-Kasai et al., 2007; Yang et al., 2008; Peng et al., 2009). Alternatively, actin filament formation could also be locally involved in PRP synthesis. Thus, it was shown that this is important for the synthesis of PKMzeta (Kelly et al., 2007), the only LTP-specific PRP characterized for CA1-LTP so far. The filaments set up by the tag of the weak stimulus might be required for local synthesis that is subsequently induced by the strong stimulus. In other words, actin filaments are necessary but not sufficient for local synthesis. However, there is also evidence that the actin network is required for other protein interactions in different hippocampal regions for LTP maintenance, i.e., in addition to PKMzeta in the CA1. For instance, in the area dentata it was shown that the synthesis of synaptopodin, an actin-associated protein is elevated during LTP maintenance (Yamazaki et al., 2001).

In summary, we provide further evidence that the "synaptic tag" consists not just of a single molecule but comprises a complex of different components (Frey and Morris, 1998a; Frey and Frey, 2008). Besides already identified process-specific tagging molecules such as CaMKII for LTP and MAPK for LTD, the actin network is actively involved in the LTP-tag-machinery in the apical CA1 dendrites. Future experiments should investigate if the actin network is also involved in late-LTD or whether it is another LTP-specific-tagging molecular complex and if it is also restricted with respect to its specific tag-action to apical CA1 dendrites (Sajikumar et al., 2007; Frey and Frey, 2008).

\section{References}

Bear MF, Malenka RC (1994) Synaptic plasticity: LTP and LTD. Curr Opin Neurobiol 4:389-399.

Chen LY, Rex CS, Casale MS, Gall CM, Lynch G (2007) Changes in synaptic morphology accompany actin signaling during LTP. J Neurosci 27:5363-5372.

Fifková E (1985) A possible mechanism of morphometric changes in dendritic spines induced by stimulation. Cell Mol Neurobiol 5:47-63.

Fischer M, Kaech S, Knutti D, Matus A (1998) Rapid actin-based plasticity in dendritic spines. Neuron 20:847-854.

Frey S, Frey JU (2008) 'Synaptic tagging' and 'cross-tagging' and related associative reinforcement processes of functional plasticity as the cellular basis for memory formation. Prog Brain Res 169:117-143.

Frey U, Morris RG (1997) Synaptic tagging and long-term potentiation. Nature 385:533-536.

Frey U, Morris RG (1998a) Synaptic tagging: implications for late maintenance of hippocampal long-term potentiation. Trends Neurosci 21:181-188.

Frey U, Morris RG (1998b) Weak before strong: dissociating synaptic tagging and plasticity- factor accounts of late-LTP. Neuropharmacology 37:545-552.

Frey U, Krug M, Reymann KG, Matthies H (1988) Anisomycin, an inhibitor of protein synthesis, blocks late phases of LTP phenomena in the hippocampal CA1 region in vitro. Brain Res 452:57-65.

Frey S, Schwiegert C, Krug M, Lössner B (1991) Long-term potentiation induced changes in protein synthesis of hippocampal subfields of freely moving rats: time-course. Biomed Biochim Acta 50:1231-1240.

Fukazawa Y, Saitoh Y, Ozawa F, Ohta Y, Mizuno K, Inokuchi K (2003) Hippocampal LTP is accompanied by enhanced F-actin content within the dendritic spine that is essential for late LTP maintenance in vivo. Neuron 38:447-460. 
Kaech S, Ludin B, Matus A (1996) Cytoskeletal plasticity in cells expressing neuronal microtubule-associated proteins. Neuron 17:1189-1199.

Kelly MT, Yao Y, Sondhi R, Sacktor TC (2007) Actin polymerization regulates the synthesis of PKMzeta in LTP. Neuropharmacology 52:41-45.

Kim CH, Lisman JE (1999) A role of actin filament in synaptic transmission and long-term potentiation. J Neurosci 19:4314-4324.

Krucker T, Siggins GR, Halpain S (2000) Dynamic actin filaments are required for stable long-term potentiation (LTP) in area CA1 of the hippocampus. Proc Natl Acad Sci U S A 97:6856-6861.

Krug M, Lössner B, Ott T (1984) Anisomycin blocks the late phase of longterm potentiation in the dentate gyrus of freely moving rats. Brain Res Bull 13:39-42.

Langford GM, Molyneaux BJ (1998) Myosin V in the brain: mutations lead to neurological defects. Brain Res Brain Res Rev 28:1-8.

Lin B, Kramár EA, Bi X, Brucher FA, Gall CM, Lynch G (2005) Theta stimulation polymerizes actin in dendritic spines of hippocampus. J Neurosci 25:2062-2069.

Ling DS, Benardo LS, Sacktor TC (2006) Protein kinase Mzeta enhances excitatory synaptic transmission by increasing the number of active postsynaptic AMPA receptors. Hippocampus 16:443-452.

Lisman J (2003) Actin's actions in LTP-induced synapse growth. Neuron 38:361-362.

Malinow R, Mainen ZF, Hayashi Y (2000) LTP mechanisms: from silence to four-lane traffic. Curr Opin Neurobiol 10:352-357.

Manahan-Vaughan D, Kulla A, Frey JU (2000) Requirement of translation but not transcription for the maintenance of long-term depression in the CA1 region of freely moving rats. J Neurosci 20:8572-8576.

Martin KC, Kosik KS (2002) Synaptic tagging - who's it? Nat Rev Neurosci 3:813-820.

Matsuzaki M, Honkura N, Ellis-Davies GC, Kasai H (2004) Structural basis of long-term potentiation in single dendritic spines. Nature 429:761-766.

Matthies H, Frey U, Reymann K, Krug M, Jork R, Schroeder H (1990) Different mechanisms and multiple stages of LTP. Adv Exp Med Biol 268:359-368.

Moshkov DA, Petrovskaia LL (1983) [Time-dependent changes in the structure of the synapses of hippocampal field CA3 after their potentiation]. Tsitologiia 25:500-507.

Nguyen PV, Kandel ER (1996) A macromolecular synthesis-dependent late phase of long-term potentiation requiring cAMP in the medial perforant pathway of rat hippocampal slices. J Neurosci 16:3189-3198.

Okamoto K, Nagai T, Miyawaki A, Hayashi Y (2004) Rapid and persistent modulation of actin dynamics regulates postsynaptic reorganization underlying bidirectional plasticity. Nat Neurosci 7:1104-1112.

Okamoto K, Narayanan R, Lee SH, Murata K, Hayashi Y (2007) The role of
CaMKII as an F-actin-bundling protein crucial for maintenance of dendritic spine structure. Proc Natl Acad Sci U S A 104:6418-6423.

Otani S, Marshall CJ, Tate WP, Goddard GV, Abraham WC (1989) Maintenance of long-term potentiation in rat dentate gyrus requires protein synthesis but not messenger RNA synthesis immediately post-tetanization. Neuroscience 28:519-526.

Pak DT, Yang S, Rudolph-Correia S, Kim E, Sheng M (2001) Regulation of dendritic spine morphology by SPAR, a PSD-95-associated RapGAP. Neuron 31:289-303.

Peng Y, Zhao J, Gu QH, Chen RQ, Xu Z, Yan JZ, Wang SH, Liu SY, Chen Z, Lu W (2009) Distinct trafficking and expression mechanisms underlie LTP and LTD of NMDA receptor-mediated synaptic responses. Hippocampus. Advance online publication. Retrieved September 10, 2009. doi: 10.1002/hipo.20654.

Sajikumar S, Navakkode S, Frey JU (2005a) Protein synthesis-dependent long-term functional plasticity: methods and techniques. Curr Opin Neurobiol 15:607-613.

Sajikumar S, Navakkode S, Sacktor TC, Frey JU (2005b) Synaptic tagging and cross-tagging: the role of protein kinase Mzeta in maintaining longterm potentiation but not long-term depression. J Neurosci 25:57505756.

Sajikumar S, Navakkode S, Frey JU (2007) Identification of compartmentand process-specific molecules required for "synaptic tagging" during long-term potentiation and long-term depression in hippocampal CA1. J Neurosci 27:5068-5080.

Serrano P, Yao Y, Sacktor TC (2005) Persistent phosphorylation by protein kinase Mzeta maintains late-phase long-term potentiation. J Neurosci 25:1979-1984.

Sheng M, Lee SH (2001) AMPA receptor trafficking and the control of synaptic transmission. Cell 105:825-828.

Shoji-Kasai Y, Ageta H, Hasegawa Y, Tsuchida K, Sugino H, Inokuchi K (2007) Activin increases the number of synaptic contacts and the length of dendritic spine necks by modulating spinal actin dynamics. J Cell Sci 120:3830-3837.

Tanaka J, Horiike Y, Matsuzaki M, Miyazaki T, Ellis-Davies GC, Kasai H (2008) Protein synthesis and neurotrophin-dependent structural plasticity of single dendritic spines. Science 319:1683-1687.

Yamazaki M, Matsuo R, Fukazawa Y, Ozawa F, Inokuchi K (2001) Regulated expression of an actin-associated protein, synaptopodin, during long-term potentiation. J Neurochem 79:192-199.

Yang Y, Wang XB, Frerking M, Zhou Q (2008) Delivery of AMPA receptors to perisynaptic sites precedes the full expression of long-term potentiation. Proc Natl Acad Sci U S A 105:11388-11393. 